Ian David Lockhart Bogle and Michael Fairweather (Editors), Proceedings of the 22nd European Symposium on Computer Aided Process Engineering, 17 - 20 June 2012, London.

(C) 2011 Elsevier B.V. All rights reserved.

\title{
Proposition of methodology for optimization of en- ergy system design under uncertainty
}

\author{
Matthias Dubuis ${ }^{\mathrm{a}}$, François Maréchal ${ }^{\mathrm{a}}$ \\ ${ }^{a}$ Industrial Energy Systems Laboratory (LENI), Ecole Polytechnique Fédérale de Lau- \\ sanne, CH-1015 Lausanne, Switzerland
}

\begin{abstract}
Current superstructure size for energy system design is increasing with computing facility improvement. Despite such approach allows to link and to analyse a lot of elements, demonstrating the advantage of a global optimization, non-negligible uncertainties are introduced. They may be due to the level of detail of modelisation, predictive aspect of the model, or simply the stochastic nature of some parameters (like outdoor temperature by example). In the present paper, different approach for optimizing energy system under uncertainty will be discussed, taking into account ability of the system to adapt to uncertain variables variations.
\end{abstract}

Keywords: process design, multi-objective optimization, uncertainty, propagation

\section{Introduction}

In current optimization of energy system design, uncertainties are often treated by considering their mean value, which shall be determine by data reconciliation techniques (Heyen et al. (1996)). However, not including uncertainties in the optimization procedure may leads to suboptimal solution that can be classified in three levels of seriousness. In best cases, performance changes will be observed due to a non-adapted control strategy or design. A worse case would be soft constraint violation, which would induce a penalty. Difference between these two cases can be describe by a heat stream to be cooled, considering uncertain temperatures. If a direct cooling system is considered, performances will change with respect to the $\Delta T_{\min } / 2$ (performances increase or decrease). If these streams temperatures are lower than the cooling system, a penalty (a refrigerating system) has to be bought. The third case, being the worst is hard constraint violation making the system unable to be operated.

In consequence, it is important to study influence of uncertainty on objectives $f(x)$ and constraints $g(x)$. This influence can be quantified by maximum variation, quantile or probability distribution function.

Several methods allow to propagate uncertainties through a model. Monte-Carlo simulation are totally based on the deterministic model. However, computing time grows exponentially with the number of uncertain variables $n_{u}$. Moreover, energy system superstructure are often non-injective. It implies that probability of having a set of uncertain variables is not the same as the probability of having the corresponding performance. Hoffmann (2001) has used orthogonal polynomials, also called Wiener-Hermite polynomial chaos expansion. Without going in the details of such method, one of the key step is estimating coefficient of polynomials (Hermite for normal distribution). This can be done by collocation method, but necessitates several calls to the deterministic model. The number of these calls is hard to determine independently of the model and then hard to implement in a generic manner. Hence perturbation method has been considered. It is already widely used in structure mechanics (Hong et al. (1999); Mekid and Vaja (2008)) and in aerodynamics (Hirsch et al. (2011)). 


\section{Problem formulation}

Globally, optimization problem under constraints are formulated as:

$$
\begin{array}{ll}
\min _{\vec{x}} & \vec{y}=f(\vec{x}) \\
\text { s.t. } & h(\vec{x})=0 \\
& g(\vec{x}) \leqslant 0
\end{array}
$$

In case of energy system design, $f(\vec{x}), h(\vec{x})$ and $g(\vec{x})$ consist in a model that will be called the deterministic model. Objectives $(f(\vec{x}))$ usually considered may be efficiency, investment cost or any environmental factor like $\mathrm{CO}_{2}$ emissions. Such model are based on flowsheeting software, or include energy integration (MILP - Mixed Integer Linear Programming). Hence, explicit function are not necessary available.

Variables $\vec{x}$ can be classified in several categories. In the first classification level, $\vec{x}$ includes decision variables $\vec{z}$ and parameters $\vec{\theta}$ with:

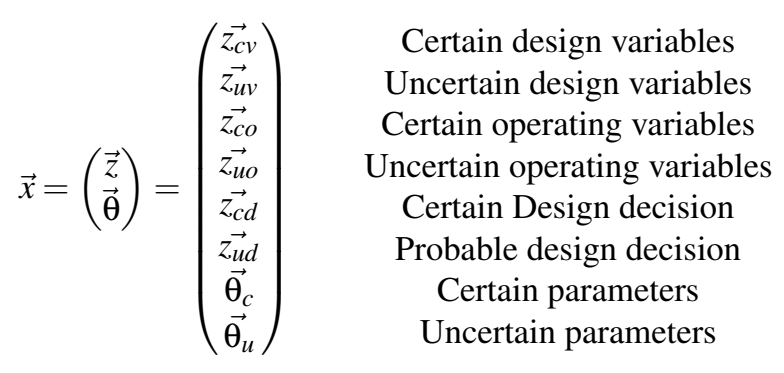

Dependent variables are not mentioned since their can be found by solving $h(\vec{x})=0$. Design variables $\overrightarrow{z_{v}}$ are continuous variables that will be fixed once the system is built. In the opposite, operating variables $\overrightarrow{z_{o}}$ are those remaining influent after installation. It should be noticed that these variables are decomposed in a set point value $\vec{z}_{u}^{*}$ and its variation $\overrightarrow{\tilde{z}}_{u}$. Design decision variables $\overrightarrow{z_{d}}$ consist only in binary variables. In that case, uncertainty is translated in a probability of the decision, as by example the probability that a household accept to be connected to a district heating. Hence, the different possibility will be treated in a multi-scenario approach. Finally, parameters are constant variables.

At this level it is considered that the different scenario related to $z_{d}$ are optimized separately, so that main issue concerns continuous uncertainties treatment. With respect to this classification, two stage programming problem as described by Grossmann et al. (1983) is expressed as:

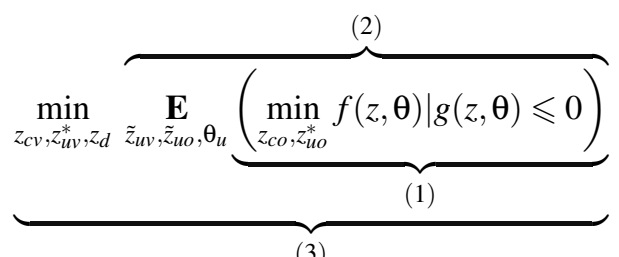

With $\mathbf{E}(x)$ being the expected value of $x$. Starting from the center, equation 3 can be described as three layer:

1. The inlet layer can be expressed as finding the best operating conditions for a given design and uncertain variables/parameters, respecting the constraints $g(z, \theta) \leqslant 0$.

2 . In the middle layer, the expected value on every uncertain variables is then considered to investigate the whole uncertainty space.

3. The outlet layer consist in the design optimization itself. 
However, this problem called infinite since operating conditions optimization is achieved for any uncertain variables configuration, which is an infinite number of problem to solve. Despite this may be solved by discretizing the uncertain variables space and solve it as a multi-period problem (Sahinidis (2004)), number of model evaluation grows exponentially with $n_{u}$. It is then crucial to apply uncertainty efficient propagation method on the model.

\section{Perturbation method}

From here one scenario will be considered (i.e. one configuration of $\vec{z}_{u d}$ ). For notation simplification other uncertain variables $\left(\vec{z}_{u v}, \vec{z}_{u o}\right.$ and $\left.\vec{\theta}_{u}\right)$ will be gathered in $\vec{u}$ and certain variables in $\vec{c}$.

Goal is to propagate uncertainties through a model by computing its characteristics, i.e. mean $\mu$, variance $\sigma$ and skewness $\gamma_{1}$. Principle is to consider Taylor series expansion of $f(\vec{u})$ for given certain variables $\vec{c}$. This series allows to define, for the mean by example as well as for other distribution parameters, $\mu_{y}$ as a function of $\mu_{u_{i}}$. The fact that Taylor series are valid only locally implies that propagation shall be computed each iteration of the optimizer.

Second order Taylor expansion in $\vec{u}=\mu$ is expressed as:

$$
y=f(\vec{u}) \cong f(\vec{u})_{\mu}+\sum_{i=1}^{n_{u}}\left(\frac{\partial f(\vec{u})}{\partial u_{i}}\right)_{\mu} \cdot\left(u_{i}-\mu_{u_{i}}\right)+\frac{1}{2} \sum_{i, j=1}^{n_{u}}\left(\frac{\partial^{2} f(\vec{u})}{\partial u_{i} \partial u_{j}}\right)_{\mu} \cdot\left(u_{i}-\mu_{u_{i}}\right) \cdot\left(u_{j}-\mu_{u_{j}}\right)
$$

So that mean and standard deviation are expressed as (Hong et al. (1999)):

$$
\begin{aligned}
\mu_{y}= & y_{\mu}+\frac{1}{2} \sum_{i=1}^{n_{u}}\left(\frac{\partial^{2} y}{\partial^{2} u_{i}}\right)_{\mu} \cdot \sigma_{u_{i}}^{2} \\
\sigma_{y}^{2}= & y_{\mu}^{2}+\sum_{i=1}^{n_{u}}\left[\left(\frac{\partial y}{\partial u_{i}}\right)^{2}+\left(y \frac{\partial^{2} y}{\partial u_{i}^{2}}\right)\right]_{\mu} \sigma_{u_{i}}^{2}+\sum_{i=1}^{n_{u}}\left(\frac{\partial y}{\partial u_{i}} \cdot \frac{\partial^{2} y}{\partial u_{i}^{2}}\right)_{\mu} \gamma_{u_{i}}-\mu_{y}^{2} \\
\gamma_{y}= & y_{\mu}^{3}+\frac{3}{2} \sum_{i=1}^{n_{u}}\left[2 y\left(\frac{\partial y}{\partial u_{i}}\right)^{2}+\left(y^{2} \frac{\partial^{2} y}{\partial u_{i}^{2}}\right)\right]_{\mu} \sigma_{u_{i}}^{2} \\
& +\sum_{i=1}^{n_{u}}\left[\left(\frac{\partial y}{\partial u_{i}}\right)^{3}+3 y \frac{\partial y}{\partial u_{i}} \frac{\partial^{2} y}{\partial u_{i}^{2}}\right]_{\mu} \gamma_{u_{i}}-\mu_{y}^{3}-3 \mu_{y} \sigma_{y}^{2}
\end{aligned}
$$

Derivative are estimated numerically at their first order:

$$
\begin{gathered}
\frac{\partial y}{\partial u_{i}}=f^{\prime}\left(u_{i}\right) \cong \lim _{h \rightarrow 0} \frac{f\left(u_{i}+h / 2\right)-f\left(u_{i}-h / 2\right)}{h} \\
\frac{\partial^{2} y}{\partial u_{i}^{2}}=f^{\prime \prime}\left(u_{i}\right) \cong \lim _{h \rightarrow 0} \frac{f\left(u_{i}+h\right)-2 f\left(u_{i}\right)+f\left(u_{i}-h\right)}{h^{2}}
\end{gathered}
$$

Skewness is considered since several parameters have great chances to be lower than what is predicted, what may leads to high asymmetry in the probability density of objective function.

It results from equation 5 that the three first moment of $f(\vec{u})$ can be computed with a number $n_{o b j}\left(2 n_{u}+1\right)$ evaluations (with $n_{o b j}$ being the number of objectives, or the size of 
¥). Compared to Monte-Carlo simulation, such approach is more competitive and enough efficient to be included in an optimization procedure, even when using method demanding a lot of iteration as evolutionary algorithm (mainly used in LENI).

However, unlike two stage programming problem (3), uncertainty propagation does not include system ability to adapt by optimizing operating decision variables.

\section{Two stage problem issues}

The following proposition is an attempt to dissociate operating decision variables from design variables. This approach requires a solver able to deal with a lot of decision variables, but does not necessitate more evaluation of the deterministic model. Principle is to decompose problem in three subproblems. As represented in figure 1, operating variables $z_{c o}$ and $z_{u o}^{*}$ may have each a different value.

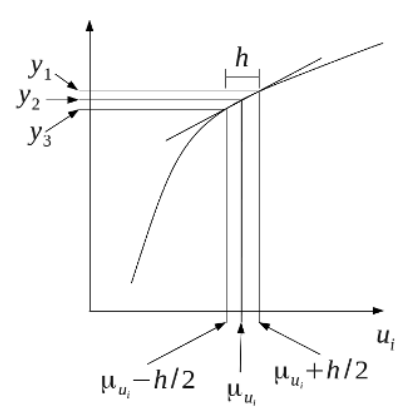

(a) Standard derivative estimation

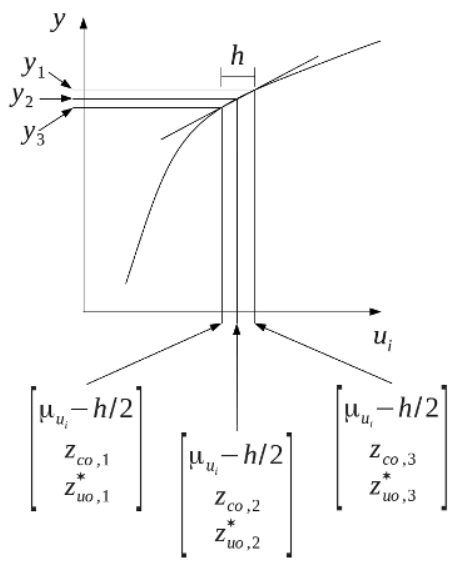

(b) "Two stage" derivative estimation

Figure 1: Derivative estimation

$$
\begin{aligned}
& \frac{\partial y}{\partial u_{i}}=f^{\prime}\left(u_{i}\right) \cong \lim _{h \rightarrow 0} \frac{f\left(u_{i}-2 h\right)-8 f\left(u_{i}-h\right)+8 f\left(u_{i}+h\right)-f\left(u_{i}+2 h\right)}{12 h} \\
& \frac{\partial^{2} y}{\partial u_{i}^{2}}=f^{\prime \prime}\left(u_{i}\right) \cong \lim _{h \rightarrow 0} \frac{-f\left(u_{i}-2 h\right)+16 f\left(u_{i}-h\right)-30 f\left(u_{i}\right)+16 f\left(u_{i}+h\right)-f\left(u_{i}+2 h\right)}{12 h}
\end{aligned}
$$

This way, ability of the plant operator to compensate uncertainties effect to maximize defined objectives. However, several issues shall be studied. First, Derivative estimation becomes strongly related to operating variables optimality. Hence, constraint shall be set so as to maintain $z_{c o, 1}$ and $z_{c o, 3}$ close to $z_{c o, 2}$, respectively $z_{u o, 1}^{*}, z_{u o, 3}^{*}$ to $z_{u o, 2}^{*}$. Secondly, It must be noticed that $h$ is a critical parameter since it has to be small enough to ensure derivative accuracy, and big enough so that optimizer may found different solution for $z_{c o, 1}, z_{c o, 3}, z_{u o, 1}^{*}$ and $z_{u o, 3}^{*}$. In case such value can not be found, several alternatives are possible but increase the number of model evaluations per iteration to $n_{o b j}\left(4 n_{u}+1\right)$. First possibility is to consider derivative with a fourth order accuracy (equation 7). In case $2 h$ remains not sufficient, derivative may be estimated based on $h=h_{1}$ for $z_{c o, 2}$ and $z_{u o, 2}^{*}$, and 
the "two stage" derivatives applied with $h=h_{2}$ and $h_{1}<<h_{2}$, interpolating the influence of operating variables on the derivative.

\section{Conclusions and perspectives}

Energy system design optimization problem are difficult to optimized since they may present several local optima. Moreover, unless the model consists in explicit function, there is no way to verify convexity and then to insure that optimality conditions are respected. As a results of this, optimizer like evolutionary algorithm may necessitate a lot of iteration to find the global optimum, what stress importance of propagating uncertainties in as less model evaluations as possible.

Several uncertainty propagation methods have been studied. Monte-Carlo simulation necessitates the biggest number of evaluation and may not be used during an optimization. However, it remains efficient for assessing solutions distribution accuracy. Polynomial chaos may be too computer resource costly due to the number of evaluations demanded for collocation. Hence, perturbation theory has been considered since it necessitates in its basic form only $n_{o b j}\left(2 n_{u}+1\right)$ deterministic model evaluation.

To reflect flexibility of the system, operating variables optimization has been included in derivative estimation. Such approach allows to include two stage optimization in parallel, solving design and corresponding operating strategy simultaneously. However, this method remains highly sensitive to $h$ parameter and increases the number of decision variables. Moreover in case of fourth order accuracy needed for the derivative, the number of model evaluation per optimization iteration increase to $n_{o b j}\left(4 n_{u}+1\right)$.

Several important issues still need to be answered. One of these is to insure feasibility (i.e hard constraint respect) for every solution. Indeed, FORM/SORM methods may be difficult to adapt since no explicit form of the constraint exist.

\section{References}

Grossmann, I. E., Halemane, K. P., and Swaney, R. 1983. Optimization strategies for flexible chemical process. Computers and Chemical Engineering, 7(4):439-462.

Heyen, G., Maréchal, E., and Kalitventzeff, B. 1996. Sensitivity calculations and variance analysis in plant measurement reconciliation. Computers and Chemical Engineering, 20(SUPPL.1):S539-S544. Cited By (since 1996): 29.

Hirsch, C., Roge, G., Karl, A., Couailler, V., Dervieux, A., Eisfeld, B., Duftoy, A., Pasanisi, A., and Hutton, A. 2011. Uncertainty management and quantification in industrial analysis and design.

Hoffmann, H. V. 2001. Multi-objective Decision Making under Uncertainty in Chemical Process Design. PhD thesis, Zurich, Switzerland.

Hong, Y. J., Xing, J., and Wang, J. B. 1999. A second-order third-moment method for calculating the reliability of fatigue. International Journal of Pressure Vessels and Piping, 76(8):567-570. Cited By (since 1996): 3.

Mekid, S. and Vaja, D. 2008. Propagation of uncertainty: Expressions of second and third order uncertainty with third and fourth moments. Measurement: Journal of the International Measurement Confederation, 41(6):600-609. Cited By (since 1996): 3.

Sahinidis, N. V. 2004. Optimization under uncertainty: State-of-the-art and opportunities. Computers and Chemical Engineering, 28(6-7):971-983. Cited By (since 1996): 170. 